\title{
Localization of delamination in composite test specimen
}

\author{
Milan Sapieta ${ }^{1, *}$, Peter $\breve{S} \operatorname{lka}^{1}$, Martin Svoboda $^{2}$ \\ ${ }^{1}$ Faculty of Mechanical Engineering, Univerzity of Žilina. Unierzitná 1, 01026 Žilina. Slovak \\ Republic \\ ${ }^{2}$ Faculty of production technology and management of Jan Evangelista Purkyně University in Ústí \\ nad Labem, Na Okraji 1001, Czech Republic
}

\begin{abstract}
The main purpose of paper is presented an innovative approach in the field of infrared thermal imaging and its integration into the area of engineering applications. As the test specimen was selected composite rod with lengthwise composite fibers to each bonded. They have been excitated with ultrasound. For obtain the results was used Lock-in method. Last part of the paper is a description of the results and most appropriate settings of measurement technique for applications with chosen material.
\end{abstract}

Keywords: infrared radiation, non-destructive testing, lock-in, ultrasound

\section{Introduction}

Infrared thermography has developed from a rarely used method to the method growing in popularity among users in recent years. This progress contributed largely by the availability and constantly falling prices of infrared cameras. For this reason, some methods have been developed which make use of infrared thermography in industry [1].

Pulse thermography was invented for the purpose of non-destructive testing (NDT) of materials and components, hence the "looking under the surface 'bodies. In pulsed thermography is pulsed heat flux generated on the surface typically by means of irradiation with light (e.g. lamp) and then is surface temperature observed in time depending. If inhomogeneity for example: cracks, holes or cavities with different temperature parameters, lies below the surface of the test object, it will have an impact on local dynamic heat flux that passes through the specimen.

As an alternative technique is offered to lock-in thermography, which is also known as heat waves imaging technique, it can be described by the theory of oscillating heat waves. Excitation of the heat occurs periodically with a certain frequency in the body [2]. The advantage of lock-in thermography is that with applying processing of the registered image it may considerably increase its sensitivity compared with the nominal sensitivity of the thermal camera.

\footnotetext{
* Corresponding author: milan.sapieta@,fstroj.uniza.sk

Reviewers: Robert Grega, Wiestawa Piekarska
} 


\section{LOCK-IN thermography}

A prerequisite for the use of this technology is that the excitation signal may be periodically pulsate or in any other way modulated by certain amplitude frequencies, called "lock-in frequency" $f_{\text {lock-in }}$. Lock-in thermography is a type of active lock-in method. It means that heat to excite the sample is generated periodically and correlation using the lock-in process is applied to all heat signatures of every single pixel on the image of the observed object.

Digital lock-in process correlation consists of averaging the results of the measured values $F_{k}$ and system of weighting factors $K_{k}$ to the total number of measured values $M$ :

$$
S=\frac{1}{M} \sum_{k=1}^{M} F_{k} K_{k}
$$

where $S$ is the output signal.

If the excitation is harmonic then the most advantageous correlation function is also harmonic (sine, cosine) function. This kind of lock-in correlation is called $\sin / \cos$ or narrowband correlation. It can be realized either by narrowing the bandwidth of the detected signal or using the values of harmonic functions for $K_{k}$ in the equation (1).

The main advantage of the $\sin / \cos$ correlation is that it enables to user to take into consideration the phase of the signal after the measurement (off-line) when it is used twochannel correlation. Two-channel correlation means that there were used two types of weighting factors, one approximates a sin-function and the second approximates cosfunction. Correlation is transferred twice in parallel with both types of weighting factors:

$$
\begin{gathered}
K^{0}(t)=2 \sin \left(2 \pi f_{\text {lock-in }} t\right), \\
K^{\pi / 2}(t)=2 \cos \left(2 \pi f_{\text {lock-in }} t\right) .
\end{gathered}
$$

Then the first channel measures the component in-phase with the sin-function, and the other channel measures the component in-phase with the cos-function, which is $\pi / 2$ phaseshifted to the sin-function [3].

If equations $(2,3)$ are inserted into equation (1) than the result of the two correlations over a complete number periods is:

$$
\begin{aligned}
S^{0} & =\frac{1}{n} \sum_{i=1}^{n} F_{i} K_{i}^{0} \\
S^{\frac{\pi}{2}} & =\frac{1}{n} \sum_{i=1}^{n} F_{i} K_{i}^{\frac{\pi}{2}}
\end{aligned}
$$

Where $S^{0}$ is called the in-phase signal and $S^{\frac{\pi}{2}}$ is usually called the quasrature signal. Both signals may be either positive or negative [4].

\section{Ultrasound excitation}

Typical set of the lock-in analysis consists of a PC, an infrared camera, an amplifier and generator lock-in frequency. It is precisely the type of generator lock-in frequency will vary different sets, so one can say that it will divided according to the type of excitation.

We decided to use ultrasonic excitation type in other measurements, which is compared with the optical excitation more suitable for steel materials, which have the largest representation in engineering practice yet. Ultrasound lock-in thermography uses the interaction between mechanical and thermal waves to detect material defects. If some damage in components absorbs the excitation of high energy ultrasound waves than it is locally heated. The resulting temperature gradient is captured by an infrared camera on the sample surface and it is subsequently dissipative energy visualized. 
This method is suitable for applications in the detection of cracks (as well as open and closed, regardless of its orientation), adhesion testing, rivets and welded joints. As with optical methods as well as ultrasound method, it is possible to detect delamination and impact damage in materials made of composite fibres.

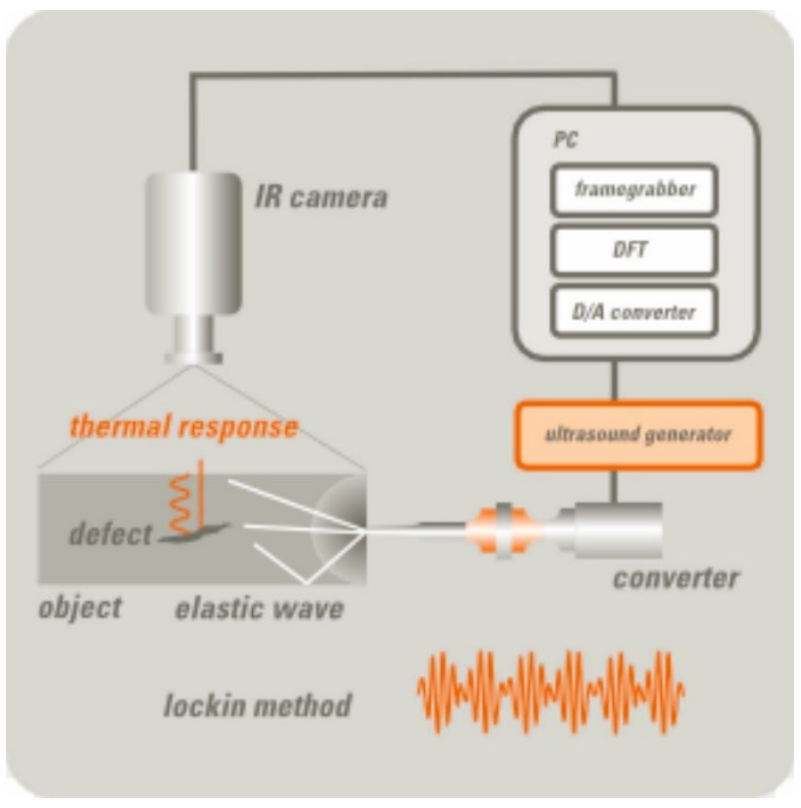

Fig. 1. Schematic representation of the lock-in principle with ultrasonic excitation Utvis.

\section{Measurement}

As the specimen was selected composite rod with longitudinally at each bonded composite fibres. The specimen was used to measure damping in the past where it was fixed in the jaws of a vice. This makes assumption of damage formation. The specimen was placed under ultrasound exciter and is subsoiled by a holder suitable shape Fig. 2.

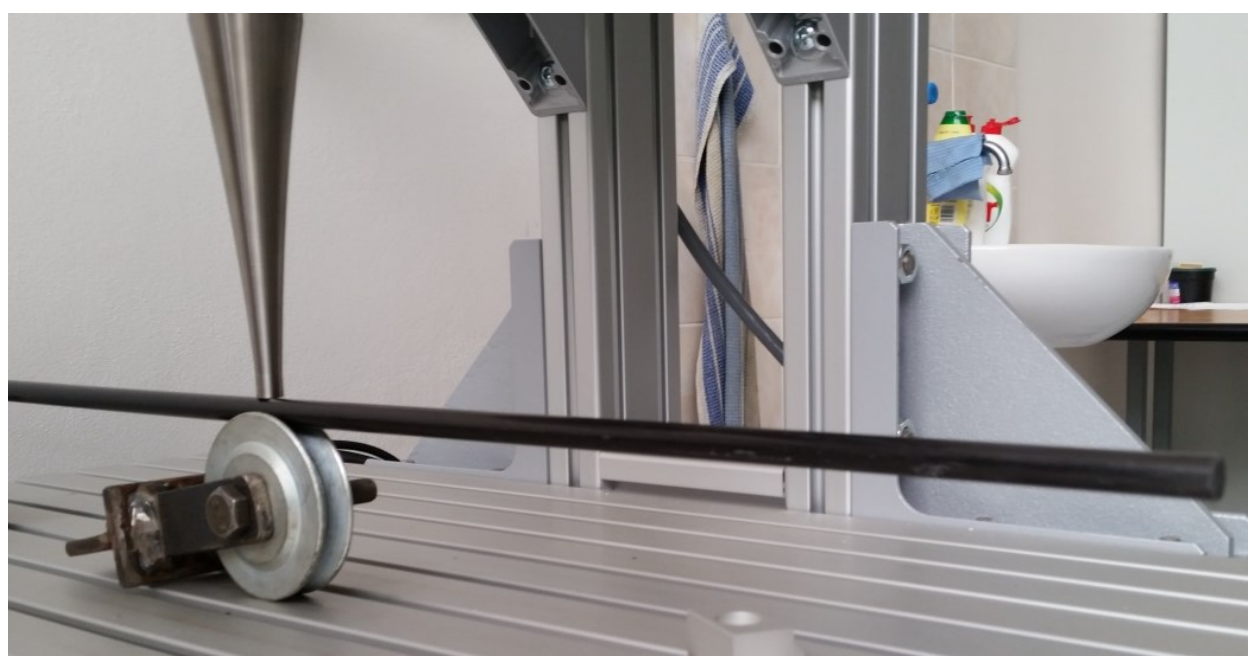

Fig. 2. Specimen placed under ultrasonic sonotrode. 
Then it was excited by selected modulated frequency until the damage was displayed. The whole process of excitation was captured by an infrared camera. Setting of IR camera was identical for each single modulation of frequency. Frame rate of IR camera was selected $50 \mathrm{~Hz}$. Value of emissivity does not affect the measurements of this type therefore it was set value 1 to the software interface. In this case the surface of the specimen is dark, so surface treatment was not necessary, as on the specimens with shiny metal surfaces, respectively coating.

The software, which was used to evaluate individual images, is called DisplayImg and it came with the excitation device. This software uses the theoretical basis of the lock-in method, as were described in the first chapter of this paper.

As a first type of excitation has been selected sweep excitation. This is excitation which passes through whole range of modulated frequencies. It is possible to observe the whole course of excitation and then choose the most appropriate modulated frequency at which is displayed defects in the measured object.

After finding the ideal modulated excitation frequency which was $0.1 \mathrm{~Hz}$, layout of radiation energy has been succeeded drawn in the software like in phase and amplitude display Fig. 4 Fig. 5. On both it is possible observed the damage in about one third of the point of excitation. This damage is more pronounced on display in-phase where it is in the form of longitudinal light lines, marked with a red ellipse Fig. 4.

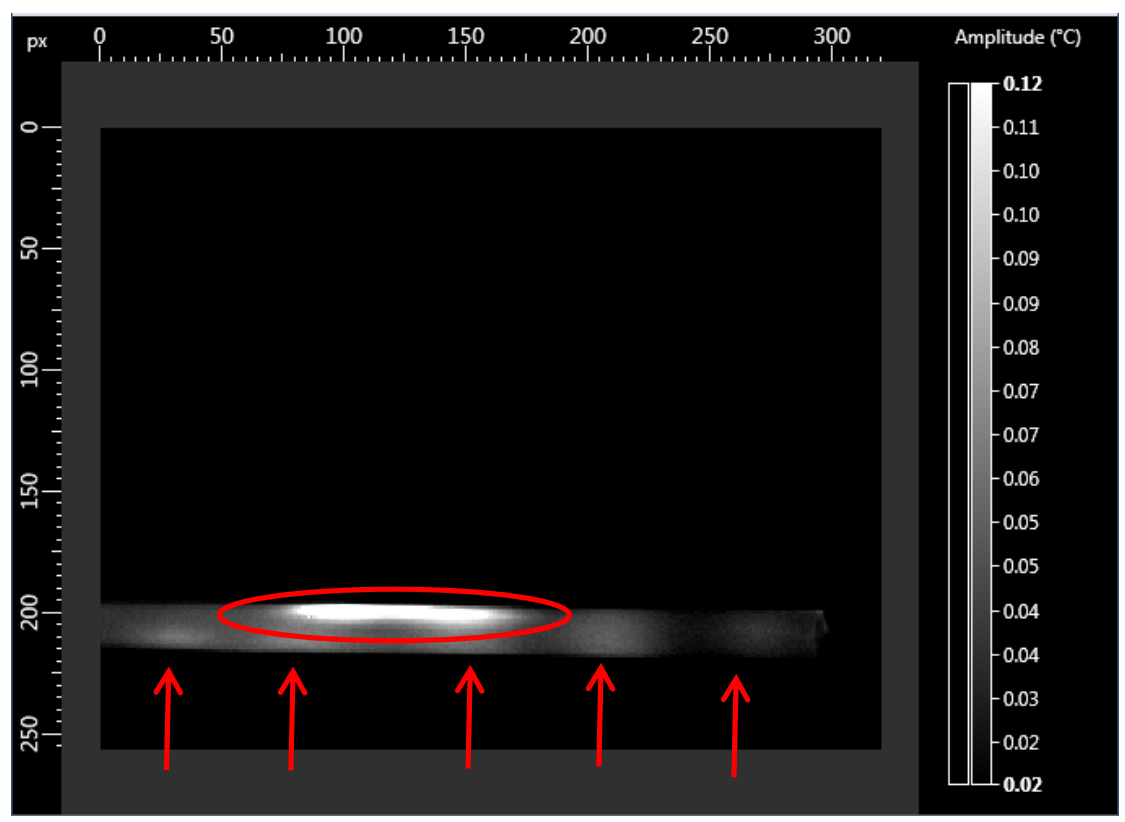

Fig. 3. Detection of damage on specimen, modulated frequency $0.1 \mathrm{~Hz}$, amplitude view, side view and damage marked with red ellipse

It can be assumed that the most likely case of delamination, separate each fiber from each other due to either the previous loading in experiments or some manufacturing defect. In Amplitude display can be seen light areas perpendicular to the fibers, marked by red arrows Fig. 3. It can be assumed that these light areas are caused by individual excitation ultrasonic waves. Therefore, it is better to evaluate the damage on the image in the displayed in-phase. 


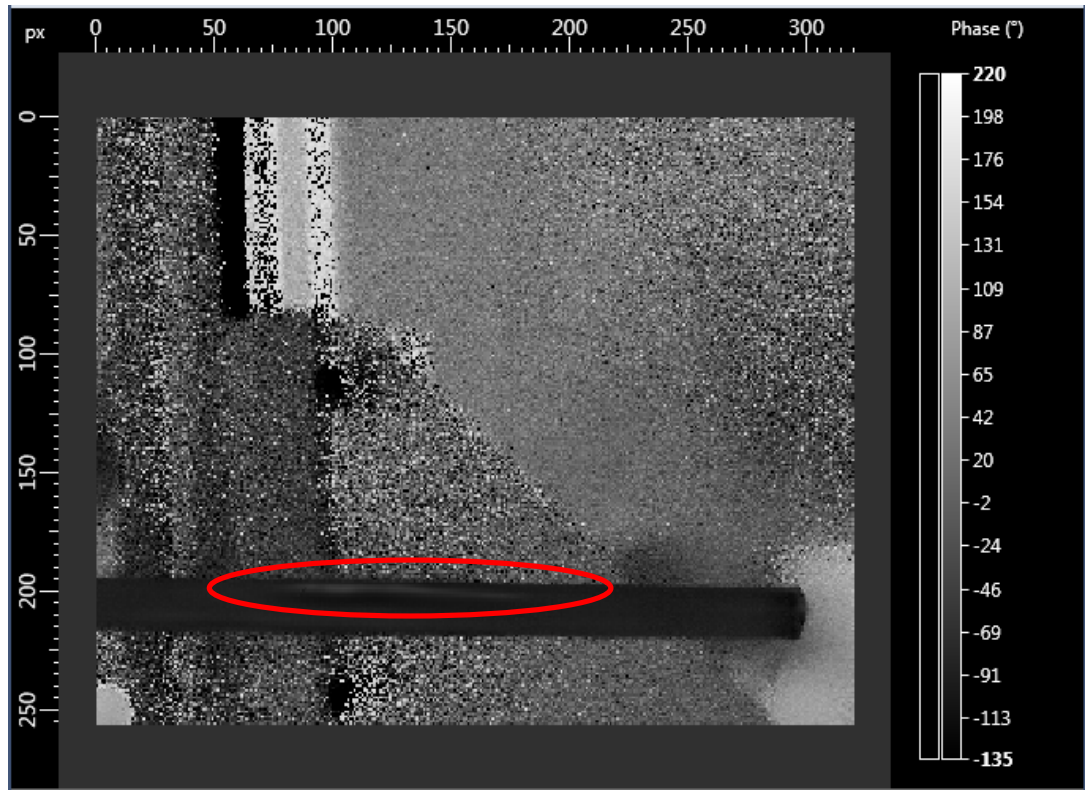

Fig. 4. Detection of damage on specimen, modulated frequency $0.1 \mathrm{~Hz}$, in-phase view, side view and damage marked with red ellipse

\section{Conclusion}

The measurement was carried out for the purpose of non-destructive testing of material. As the specimen was selected composite rod with longitudinal fibres bonded to each other with damage, which was made by either the manufactured or previous used. Using a measuring system UTvis, a device with an ultrasonic excitation used by the lock-in method for NDT has been made to test of this specimen. First it was performed ultrasonic excitation across the whole range of modulated frequency, by this excitation was chosen modulated frequency at which was able detect cracks on the side of the specimen.

For significant knowledge can be considered the fact that, as of oscillation (when changing the excitation field frequency, then if the exciting frequency coincides with its own than is a registered resonances) and in this type of excitation (ultrasound) is a response in the IR area dependents on immediate parameters of the excitation signal [5-7].

Crack was formulated by the light areas by changing the radiation layout on interpreted images. This method has proven to be a suitable solution for NDT testing to detect delamination and damage to composite fibres.

The part of the results of this work has been supported by VEGA grant No. 1/0795/16 a KEGA No. $017 \check{Z} U-4 / 2017$.

\section{References}

1. M. Sapieta, A. Sapietová, V. Dekýš, A detection of deformation mechanisms using infrared thermography and acoustic emission. Applied Mechanics and Materials 474, 315-320 (2014)

2. V. Vavilov, Thermal/Infrared testing. Nondestructive testing, Publishing house Spektr., 732 p. (2009) 
3. X. Xian, W Zheng, W. HU, Evaluation of the Damage of Carbon/Epoxy Composite Laminates under Static and Fatigue Loading by Thermographic Analyses.Proceedings of the International Symposium on Composite Materials and Structures, 710-716 (1986)

4. M. Handrik, P. Kopas, V. Baniari, M. Vaško, M. Sága, Analysis of stress and strain of fatigue specimens localised in the cross-sectional area of the gauge section testing on bi-axial fatigue machine loaded in the high-cycle fatigue region. Procedia Engineering 177, 516-519 (2017)

5. P. Pecháč, M. Sága, Controlling of local search methods' parameters in memetic algorithms using the principles of simulated annealing. Proc. Eng. 136, 70-76 (2016)

6. L. Jakubovičová, M. Sága, Computational analysis of contact stress distribution in the case of mutual stewing of roller bearing rings. Novel Trends in Production Devices and Systems, Applied Mechanics and Materials 474, 363-368 (2014)

7. J. Zapoměl, V. Dekýš, P. Ferfecki, A. Sapietová, M. Sága, M. Žmindák, Identification of material damping of a carbon composite bar and study of its effect on attenuation of its transient lateral vibrations. Int. Journal of Applied Mechanics 7 (6), (2015) 